\title{
Thermal effects versus spin nematicity in a frustrated spin- $\frac{1}{2}$ chain
}

\author{
M. Pregelj $\odot,{ }^{1, *}$ A. Zorko $\odot,{ }^{1,2}$ D. Arčon $\odot,{ }^{1,2}$ M. Klanjšek $\odot,{ }^{1}$ O. Zaharko, ${ }^{3}$ S. Krämer $\odot,{ }^{4}$ M. Horvatić $\odot,{ }^{4}$ and A. Prokofiev $\oplus^{5}$ \\ ${ }^{1}$ Jožef Stefan Institute, Jamova c. 39, 1000 Ljubljana, Slovenia \\ ${ }^{2}$ Faculty of Mathematics and Physics, University of Ljubljana, Jadranska u. 19, 1000 Ljubljana, Slovenia \\ ${ }^{3}$ Laboratory for Neutron Scattering and Imaging, PSI, CH-5232 Villigen, Switzerland \\ ${ }^{4}$ Laboratoire National des Champs Magnétiques Intenses, LNCMI-CNRS (UPR3228), EMFL, Université Grenoble Alpes, \\ UPS and INSA Toulouse, Boîte Postale 166, 38042 Grenoble Cedex 9, France \\ ${ }^{5}$ Institute of Solid State Physics, Vienna University of Technology, Wiedner Hauptstrasse 8-10, 1040 Vienna, Austria
}

(Received 13 December 2019; revised 8 June 2020; accepted 20 July 2020; published 5 August 2020)

\begin{abstract}
The spin-nematic phase is an intriguing state of matter that lacks usual long-range dipolar order, yet it exhibits higher multipolar order. This makes its detection extremely difficult and controversial. Recently, nuclear magnetic resonance (NMR) has been proposed as one of the most suitable techniques to confirm its existence. We report a ${ }^{17} \mathrm{O} N M R$ observation of the reduction of the local magnetization in the polarized state of the frustrated spin- $\frac{1}{2}$ chain $\beta-\mathrm{TeVO}_{4}$, which was previously proposed to be a fingerprint of the spin-nematic behavior. However, our detailed study shows that the detected missing fraction of the magnetization, probed by NMR frequency shift, is thermally activated, thus undermining the presence of the spin-nematic phase in the investigated compound. This highlights the importance of careful considerations of temperature-dependent NMR shift that has been overlooked in previous studies of spin nematicity.
\end{abstract}

DOI: 10.1103/PhysRevB.102.081104

The pursuit of a spin-nematic phase [1,2] has been attracting physicists for almost half a century [1-8]. In this enigmatic phase, a spin system develops long-range order of magnetic quadrupoles [1] that lacks dipolar correlations. Consequently, the spin-nematic phase is elusive and extremely difficult to detect experimentally. Lately, the research has focused on frustrated spin- $-\frac{1}{2}$ chains, where a spin-nematic phase is predicted to occur in a narrow magnetic-field range just before the magnetization saturates $[9,10]$. Here, the magnetic quadrupoles are expected to form out of bound magnon pairs that condense at the bonds between the neighboring spins $[5,11,12]$. The resulting spin-nematic order has been theoretically suggested to be detectable either directly, by probing its excitations, i.e., by breaking bound magnon pairs [13-17], or indirectly, by following the corresponding missing fraction of the magnetization [12]. Experimentally, nuclear magnetic resonance (NMR) has been proposed as the most suitable technique to detect the missing fraction due to its high resolution, allowing it to detect minute changes in local magnetization $[18,19]$. Yet, rounding of the magnetic-field-driven transition into the fully polarized spin state is characteristic also of thermal fluctuations [20,21], which have been overlooked in the previous studies of the spin nematicity $[18,19]$.

The most studied frustrated spin- $\frac{1}{2}$ chain candidate $\mathrm{LiCuVO}_{4}$ has the saturation field between 40 and $50 \mathrm{~T}$ [22]. Its magnetic response in the vicinity of the saturation has been, therefore, investigated primarily by pulsed-fieldmagnetization and nuclear-magnetic-resonance (NMR) measurements $[18,19,22]$. The latest NMR experiments revealed that at $1.3 \mathrm{~K}$ the magnetic order vanishes already $\sim 1 \mathrm{~T}$

*matej.pregelj@ijs.si before the full saturation is achieved, which was proposed as an experimental proof of the spin-nematic phase [19]. If the observed response is indeed associated with the emergence of the spin-nematic phase, then it should not change upon cooling and should persist to the lowest accessible temperatures [12], which, however, is yet to be confirmed experimentally.

Here we focus on $\beta-\mathrm{TeVO}_{4}[23,24]$, which also exhibits all the characteristics of the spin- $\frac{1}{2}$ frustrated ferromagnetic chain [25-29]. The $\mathrm{V}^{4+}\left(S=\frac{1}{2}\right)$ magnetic ions are coupled by ferromagnetic nearest-neighbor $J_{1} \approx-38 \mathrm{~K}$ and antiferromagnetic next-nearest-neighbor $J_{2} \approx-J_{1}$ exchange interactions. The system exhibits a long-range magnetic order below $T_{N 1}=4.6 \mathrm{~K}$ and the experimentally derived phase diagram resembles the theoretically predicted one very well [26]. In particular, the helical ground state is at $\sim 3 \mathrm{~T}$ succeeded by the spin-density-wave (SDW) state, which is at $\sim 18.7 \mathrm{~T}$ followed by the high-field (HF) phase with an unknown type of incommensurate magnetic order [30]. Finally, the saturation is reached at $\sim 21.7 \mathrm{~T}$ [30], allowing for a spin-nematic phase to exist in a narrow interval around this field, as theoretically predicted [31]. A relatively low saturation field compared to $\mathrm{LiCuVO}_{4}$ [22] and other related systems allows for a much more detailed NMR study of the putative spin-nematic phase, which could focus also on thermal effects.

In this work we present low-temperature high-magneticfield NMR measurements on $\beta-\mathrm{TeVO}_{4}$, focusing on the magnetic-field region where the spin-nematic phase could exist. Our data show that, between 21.72(1) and 21.78(1) T, the NMR spectrum progressively changes from a complex one, corresponding to the long-range ordered HF phase, to a simple paramagnetic one, indicative of the fully polarized, 

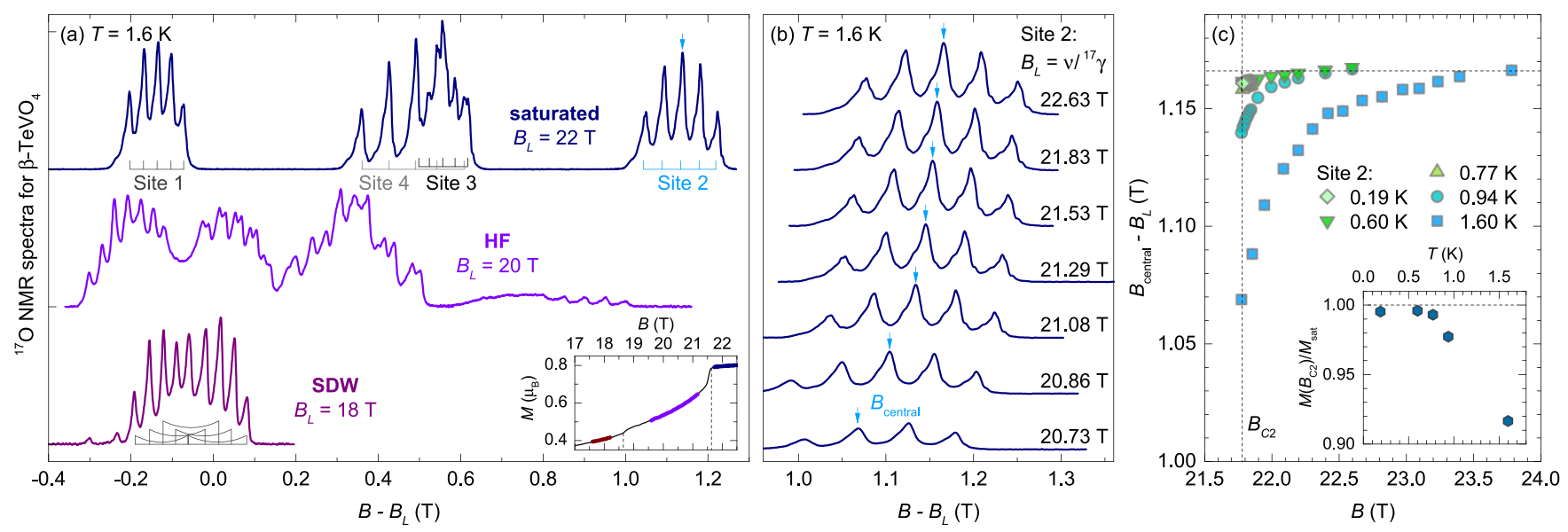

FIG. 1. The magnetic-field ( $\mathbf{B} \| a$ ) dependence of the ${ }^{17} \mathrm{O}$ NMR spectra. (a) Spectra measured by sweeping the magnetic field at fixed frequencies $v={ }^{17} \gamma B_{L}$. The forks at the baseline of the $B_{L}=22 \mathrm{~T}$ curve indicate positions of the spectral peaks (quintets), corresponding to each oxygen site. At the baseline of the $B_{L}=18 \mathrm{~T}$ spectrum U-shaped incommensurate broadening, anticipated for the SDW state, is demonstrated. Inset shows the field dependence of magnetization measured at $1.7 \mathrm{~K}$ [30] with color-coded field ranges covered by NMR spectra. (b) Detailed field dependence of the spectrum for site 2. Arrows denote the position of the central transition $B_{\text {central }}$. (c) The magnetic field dependence of the shift of the central transitions $B_{\text {central }}$ with respect to $B_{L}$ for site 2 measured at several temperatures (symbols). The dashed horizontal line denotes the value ascribed to the fully saturated magnetization $M_{\text {sat }}$. Inset shows the ratio between the magnetization at $B_{C 2}$ and $M_{\text {sat }}$ derived from the internal fields at $B_{C 2}$ and $23.8 \mathrm{~T}$, respectively.

i.e., saturated, spin state. This is characteristic of a first-order transition, where two phases coexist over a finite range of the external magnetic field. Remarkably, above $21.78 \mathrm{~T}$ the shift of the fully polarized spectrum relative to the Larmor frequency still grows with increasing field at $1.6 \mathrm{~K}$, while its width does not change anymore. This resembles the response reported for $\mathrm{LiCuVO}_{4}$ [19] and may thus imply the presence of the spin-nematic phase. However, with decreasing temperature, this shift growth becomes less and less pronounced and is finally completely suppressed below $\sim 0.8 \mathrm{~K}$. This reveals that the field-induced shift of the NMR spectrum just below the saturation in $\beta-\mathrm{TeVO}_{4}$ is not a sign of the spin-nematic phase, but is rather due to thermal fluctuations. A precise investigation of the thermal effects on the NMR shift is, therefore, clearly an essential step in the search for the spinnematic state. As such, our discovery provides an alternative explanation of the spin-nematic-like behavior that should be considered also in $\mathrm{LiCuVO}_{4}$.

A single crystal $\left(1.00 \times 3.10 \times 7.55 \mathrm{~mm}^{3}\right)$ of $\beta-\mathrm{TeVO}_{4}$ was grown from $\mathrm{TeO}_{2}$ and $\mathrm{VO}_{2}$ powders by chemical vapor transport reaction, using a two-zone furnace and $\mathrm{TeCl}_{4}$ as a transport agent $[26,28,29]$. The ${ }^{17} \mathrm{O}$ enrichment of $\sim 8 \%$ was achieved [32]. The ${ }^{17} \mathrm{O}$ NMR was measured using a custom-built spectrometer at the National Laboratory for High Magnetic Fields (LNCMI), Grenoble, France, between 0.2 and $17.7 \mathrm{~K}$ in the magnetic field $B$ between 17 and $24 \mathrm{~T}$ applied along the crystallographic $a$ axis. A dilution refrigerator was used to achieve temperatures below $1.6 \mathrm{~K}$. The spectra were measured at fixed frequencies by sweeping the magnetic field or at fixed fields by sweeping the frequency. The shape of the coil was adopted to fit the crystal (flat elongated plate), while a rotator was used to adjust the orientation of the magnetic field in the $a b$ plane. Internal capacitors (bottom tuning) were used to improve signal-tonoise ratio for temperatures above $1.6 \mathrm{~K}$, while external capacitors (top tuning) were used in combination with the dilution refrigerator.

To explore the high-field magnetic phases in $\beta$ - $\mathrm{TeVO}_{4}$ we first measured the ${ }^{17} \mathrm{O}$ NMR spectrum at $1.6 \mathrm{~K}$ in the SDW, HF, and saturated phases, i.e., at $\sim 18,20$, and $22 \mathrm{~T}$, respectively [Fig. 1(a)]. For a single ${ }^{17} \mathrm{O}$ site (nuclear spin $I=\frac{5}{2}$ ) one expects a spectrum composed of five lines (a quintet), due to the splitting of the $I=\frac{5}{2}$ multiplet in an electric field gradient present in the crystal. Indeed, in the saturated phase, the spectrum exhibits four quintets, i.e., two well separated and two overlapping quintets, corresponding to four crystallographically inequivalent oxygen sites. Relative to the "Larmor" field $B_{L}=v /{ }^{17} \gamma$, site 1 is shifted to negative fields by $0.14 \mathrm{~T}$, whereas sites $2-4$ are shifted to positive fields by $1.14,0.52$, and $0.49 \mathrm{~T}$, respectively. The shift of site 1 is notably smaller compared to the others, which indicates that the corresponding hyperfine coupling is significantly weaker. This is the reason why only spectral lines from site 1 can be detected in the SDW phase, where the signal from other sites is suppressed by fast magnetic relaxation driven by stronger hyperfine interactions. Since in the SDW phase each of the five NMR transitions is split into a characteristic Ushaped spectrum by incommensurately modulated local magnetic fields, the SDW spectrum consists of five overlapping U-shaped spectral lines [sketched in Fig. 1(a)]. In the HF phase, the spectrum is significantly broader and more complex. In particular, besides the changes of the magnetic order, we find that in this phase also sites 2-4 contribute to the spectrum, indicating that the corresponding magnetic relaxations are slower than in the SDW phase. The spectrum is completely different than in the saturated phase, implying that the local fields are still continuously distributed, as expected for an incommensurate magnetic order.

In order to investigate the potential spin-nematic behavior, we performed detailed measurements of the NMR spectrum 
as a function of the applied magnetic field in the vicinity of the magnetization saturation. Since the local magnetic field is a sum of the applied field and magnetization-driven internal fields, the shifts of the NMR lines relative to $B_{L}$ continue to change until the magnetization is fully developed. This effect is most pronounced for site 2 because it experiences the largest internal field. At $1.6 \mathrm{~K}$, we find that between $B_{C 2}=21.78(1)$ and 23.4(1) T, i.e., where the spectrum already adopts a simple saturated shape, the shift of the NMR spectrum of this site relative to $B_{L}$ still increases by an additional $0.1 \mathrm{~T}$ [Fig. 1(b)]. This becomes even clearer when plotting the shift of the central NMR transition $B_{\text {central }}$ relative to $B_{L}$ as a function of the applied magnetic field [Fig. 1(c)] [32]. A physical interpretation of this result is that, above $B_{C 2}$, the magnetization is still increasing with increasing field. The observed increase of the $\left(B_{\text {central }}-B_{L}\right)$ shift above $B_{C 2}$ could thus be a signature of the spin-nematic order, as suggested before [19].

To explore the origin of the magnetic-field-induced shift of the fully polarized spectrum and its potential relation to the spin-nematic order, we performed detailed field-dependent measurements of the central line for site 2 at several temperatures. The results, summarized in Fig. 1(c), clearly show that on cooling the corresponding internal field $\left(B_{\text {central }}-B_{L}\right)$ approaches a field-independent constant, set by the fully saturated magnetization reached at $\sim 23.8 \mathrm{~T}$. Such "rounding" of the magnetic-field-driven transition into the fully polarized spin state, whose sharpness changes with temperature, is obviously due to thermal fluctuations [20,21]. In fact, since internal fields are in the saturated phase directly proportional to the sample's magnetization, we can plot the ratio between the magnetization at $B_{C 2}$ and its fully saturated value [inset in Fig. 1(c)], which clearly shows that below $\sim 0.6 \mathrm{~K}$ the magnetization is already fully developed at $B_{C 2}$. In contrast, the spin-nematic phase in frustrated spin- $\frac{1}{2}$ chains is for $J_{1} / J_{2} \sim-1$ predicted to emerge already at zero temperature when magnetization surpasses $\sim 70 \%$ of its fully saturated value $[9,10]$, i.e., spanning across a sizable magnetization range that should not be suppressed on cooling. The observed behavior, therefore, indicates that the increase of the internal field above $B_{C 2}$ is caused by thermal fluctuations rather than being a signature of the spin-nematic order.

A detailed inspection of the transition from the HF state to the saturated state at $0.6 \mathrm{~K}$ reveals another surprising feature. Namely, across the transition, the NMR spectrum at site 2 is very complex and corresponds to the HF state coexisting with a simple (paramagneticlike) fully polarized, i.e., saturated, state [Fig. 2(a)]. On increasing field, the intensity of the fully polarized spectrum [Fig. 2(b)] becomes observable at $B_{C 1}=21.72(1) \mathrm{T}$ and gradually increases up to $B_{C 2}$, beyond which field it does not change anymore. On the contrary, the HF signal starts to lose intensity at $B_{C 1}$ and completely disappears at $B_{C 2}$ [Fig. 2(b)]. Moreover, below $B_{C 1}$ and above $B_{C 2}$ the positions of the HF and the fully polarized spectra, respectively, shift linearly with the magnetic field, while throughout the transition they do not change at all [Fig. 2(c)]. Such behavior is characteristic of an intermediate mixedphase state that develops at the first-order transition between two states with different magnetizations [33-36], indicating that the transition from the HF state to the saturated state
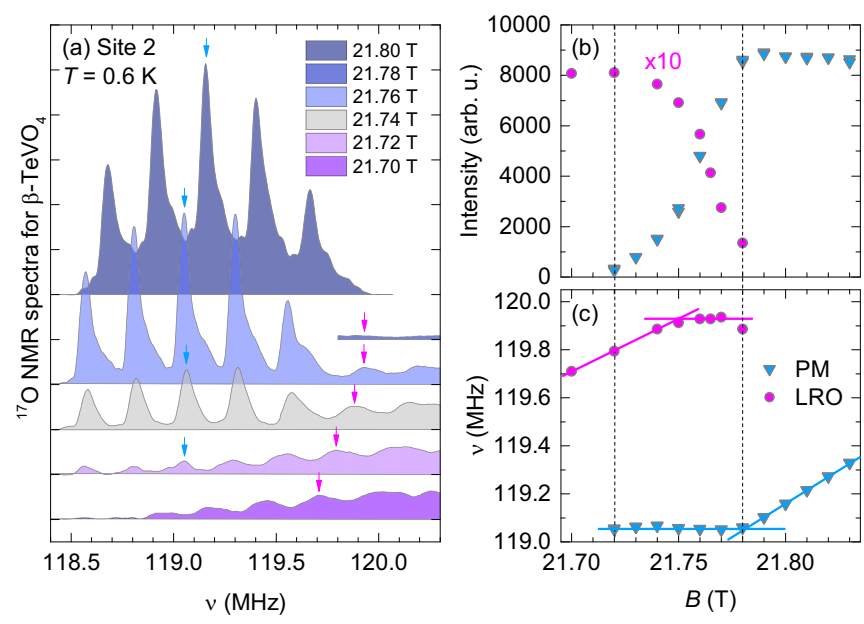

FIG. 2. (a) Frequency-dependent ${ }^{17} \mathrm{O}$ NMR spectra taken at fixed fields at the transition from the $\mathrm{HF}$ to the fully polarized state measured at $0.6 \mathrm{~K}$. The field dependencies of (b) intensity and (c) position of the spectral features indicated by arrows in panel (a). Solid lines are guides to the eye.

is a first-order type of the phase transition. In particular, the effect of the demagnetizing field makes the local field in the mixed-phase state independent of the applied field [33], which does not leave any room for a potential spin-nematic phase.

After exploring static properties of the system, we continue with our investigation of spin dynamics by utilizing the nuclear spin-lattice-relaxation $\left(1 / T_{1}\right)$ measurements. We focus our attention on the saturated state (above $B_{C 2}$ ), since in frustrated spin- $-\frac{1}{2}$ chains the exotic bound-magnon excitations are expected to be more favorable than conventional singlemagnon excitations [12]. We measured $1 / T_{1}$ at the peak of the central transition of site 2 as a function of magnetic field and temperature. The data can be nicely fitted with the expression for central transition for $I=\frac{5}{2}$ nucleus [37] with a stretching exponent of 0.80 (3) [32,38], indicating a finite distribution of $1 / T_{1}$. The derived dependence of $1 / T_{1}$ [Fig. 3(a)] exhibits a thermally activated behavior that can be described by the Arrhenius law,

$$
1 / T_{1}=1 / T_{1}^{\mathrm{HT}} \exp \left(-\Delta / k_{B} T\right),
$$

where $1 / T_{1}^{\mathrm{HT}}$ is the high-temperature limit for $1 / T_{1}, \Delta$ denotes the excitation gap, and $k_{B}$ is the Boltzmann constant. Indeed, with a field-independent value $1 / T_{1}^{\mathrm{HT}}=1.30(1) \mathrm{ms}^{-1}$, we obtain a very good agreement with the experimental data in all magnetic fields [solid lines in Fig. 3(a)]. The derived $\Delta(B)$ is a linear function of $B$ [Fig. 3(b)]. This complies with the theory $[12,39]$, predicting that at the critical field $B_{\Delta}$ the bound magnon pairs, constituting the spin-nematic state, give way to the fully saturated state and develop a gap that is proportional to the applied magnetic field. Namely, above the saturation the excitation gap should follow the $\Delta=$ $p g \mu_{B}\left(B-B_{\Delta}\right)$ dependence [18,40], where $g=2.01$ [26] is the gyromagnetic ratio and $p$ is the number of bound magnons. As expected, the field dependence of $\Delta$ can be described perfectly with $B_{\Delta}=19.91(2) \mathrm{T}$ and $p=1.99(1)$ [solid line in Fig. 3(b)], implying that above the saturation the relaxation process is dominated by two-magnon excitations. For 

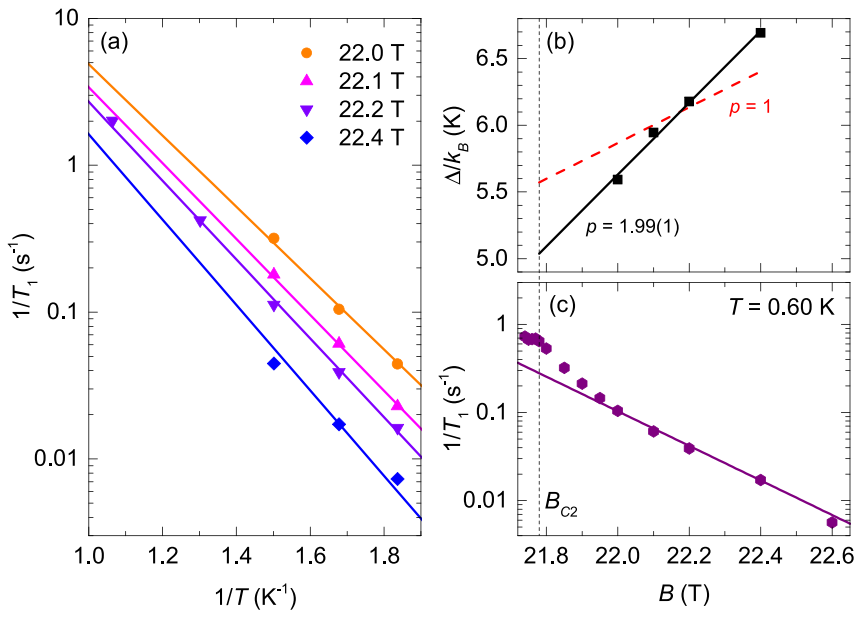

FIG. 3. (a) The ${ }^{17} \mathrm{O}$ spin-lattice-relaxation rate measured at several magnetic fields in the saturated phase at the central transition of site 2 as a function of the inverse temperature. Solid lines are fits to Eq. (1) (see text). (b) The derived excitation gap as a function of the applied magnetic field. Solid line is a linear fit, yielding two magnon excitations $(p=2)$, which is compared to a fit assuming one-magnon excitations $(p=1)$. (c) $1 / T_{1}$ measured at $0.6 \mathrm{~K}$ as a function of the applied magnetic field.

comparison we show a fit assuming one-magnon excitations, which is clearly inadequate [dashed line in Fig. 3(b)].

However, the derived parameter $B_{\Delta}$ is smaller than $B_{C 1}$, hence, the excitations appear to have a finite gap, $\Delta_{\text {sat }} / k_{B} \approx$ $5 \mathrm{~K}$, already at $B_{C 2}$, where the saturated phase is established, in line with the first-order nature of the transition. Moreover, the derived $\Delta_{\text {sat }}$ is of the order of the exchange anisotropy in the investigated system, $\delta_{1,2}^{b} / k_{B} \sim\left|0.2 J_{1,2}\right| / k_{B} \sim 7.6 \mathrm{~K}$ [29] and complies with the magnon gap of $\sim 7 \mathrm{~K}(0.6 \mathrm{meV})$ determined by inelastic neutron scattering (see Supplementary Information to Ref. [41]). It thus seems that the exchange anisotropy might be associated with the establishment of the incommensurate HF state instead of the spin-nematic one, as suggested earlier [30]. This is also in agreement with theoretical calculations, showing that the easy-plane exchange anisotropy and finite interchain interactions, which in $\beta$ - $\mathrm{TeVO}_{4}$ amount to $\sim 0.13\left|J_{1}\right|$ and $\sim 0.05\left|J_{1}\right|$, respectively [29], suppress the condensation of bound magnon pairs $[42,43]$ and thus preclude the formation of the spin-nematic phase. Last, we note that a small deviation from the two-magnon behavior is observed in $1 / T_{1}$ at $0.6 \mathrm{~K}$ [Fig. 3(c)] already below $\sim 21.95 \mathrm{~T}$, which is far above $B_{\Delta}$ where the two-magnon spin gap is expected to close, suggesting that it cannot be associated with the spin-nematic fluctuations.

Our results particularly highlight the effect of the thermal spin fluctuations. At finite temperature, the latter push the saturation of magnetization well beyond the zero-temperature limit of 21.78(1) $\mathrm{T}$, which can be mistaken for a fingerprint of the spin-nematic order. In fact, thermal spin fluctuations could also explain the contradictory reports about $\mathrm{LiCuVO}_{4}$, where NMR results for $\mathbf{B} \| c$ and $T=0.38 \mathrm{~K}$ [18] show that the magnetization is completely saturated at $\sim 42 \mathrm{~T}$, whereas the magnetization [18] and NMR [19] data obtained at $1.3 \mathrm{~K}$ exhibit a gradual increase of magnetization up to $\sim 44 \mathrm{~T}$. The observed behavior was associated either with nonmagnetic defects [18] or with spin-nematic ordering [19], but it may well be a result of thermal spin fluctuations. Hence, in order to unambiguously confirm the presence of the spin-nematic state in $\mathrm{LiCuVO}_{4}$, an in-depth temperature-dependent investigation at the lowest experimentally accessible temperatures, like ours, is required.

In conclusion, our high-magnetic-field ${ }^{17} \mathrm{O}$ NMR measurements on $\beta-\mathrm{TeVO}_{4}$ show that below $\sim 1 \mathrm{~K}$ the system undergoes a first-order transition from the HF to the fully polarized, i.e., saturated, phase at $\sim 21.75 \mathrm{~T}$. At lowest temperatures the transition extends across a narrow magnetic-field range, which manifests in an intermediate mixed-phase state, where the incommensurate HF and the fully polarized states coexist. At elevated temperatures, however, we find that the complete saturation of the magnetization is achieved only at much higher fields, which reflects the effect of thermal fluctuations. Such a response can easily be mistaken for a spin-nematic behavior potentially undermining the existing reports of spin-nematic phases in other systems. Our study thus shows that a firm confirmation of the spin-nematic state requires a careful investigation of thermal effects, a crucial experimental step that was not considered in previous studies.

This work has been funded by the Slovenian Research Agency (projects J1-9145 and N1-0148, and program No. P1-0125) and the Swiss National Science Foundation (project SCOPES IZ73Z0_152734/1). We acknowledge the support of the LNCMI-CNRS, member of the European Magnetic Field Laboratory (EMFL).
[1] A. F. Andreev and I. A. Grishchuk, Spin nematics, Zh. Eksp. Teor. Fiz. 87, 467 (1984) [Sov. Phys. JETP 60, 267 (1984)].

[2] C. Lacroix, P. Mendels, and F. Mila, Introduction to Frustrated Magnetism: Materials, Experiments, Theory, Springer Series in Solid-State Sciences (Springer, Berlin, Heidelberg, 2011).

[3] M. Blume and Y. Y. Hsieh, Biquadratic exchange and quadrupolar ordering, J. Appl. Phys. 40, 1249 (1969).

[4] P. Chandra and P. Coleman, Quantum Spin Nematics: MomentFree Magnetism, Phys. Rev. Lett. 66, 100 (1991).

[5] N. Shannon, T. Momoi, and P. Sindzingre, Nematic Order in Square Lattice Frustrated Ferromagnets, Phys. Rev. Lett. 96, 027213 (2006).
[6] M. Mourigal, M. Enderle, B. Fåk, R. K. Kremer, J. M. Law, A. Schneidewind, A. Hiess, and A. Prokofiev, Evidence of a Bond-Nematic Phase in $\mathrm{LiCuVO}_{4}$, Phys. Rev. Lett. 109, 027203 (2012).

[7] J. A. M. Paddison, H. Jacobsen, O. A. Petrenko, M. T. Fernández-Díaz, P. P. Deen, and A. L. Goodwin, Hidden order in spin-liquid $\mathrm{Gd}_{3} \mathrm{Ga}_{5} \mathrm{O}_{12}$, Science 350, 179 (2015).

[8] Y. Kohama, H. Ishikawa, A. Matsuo, K. Kindo, N. Shannon, and Z. Hiroi, Possible observation of quantum spin-nematic phase in a frustrated magnet, Proc. Natl. Acad. Sci. USA 116, 10686 (2019). 
[9] T. Hikihara, L. Kecke, T. Momoi, and A. Furusaki, Vector chiral and multipolar orders in the spin- $\frac{1}{2}$ frustrated ferromagnetic chain in magnetic field, Phys. Rev. B 78, 144404 (2008).

[10] J. Sudan, A. Lüscher, and A. M. Läuchli, Emergent multipolar spin correlations in a fluctuating spiral: The frustrated ferromagnetic spin- $\frac{1}{2}$ Heisenberg chain in a magnetic field, Phys. Rev. B 80, 140402(R) (2009).

[11] A. V. Chubukov, Chiral, nematic, and dimer states in quantum spin chains, Phys. Rev. B 44, 4693 (1991).

[12] M. E. Zhitomirsky and H. Tsunetsugu, Magnon pairing in quantum spin nematic, Europhys. Lett. 92, 37001 (2010).

[13] M. Sato, T. Momoi, and A. Furusaki, NMR relaxation rate and dynamical structure factors in nematic and multipolar liquids of frustrated spin chains under magnetic fields, Phys. Rev. B 79, 060406(R) (2009).

[14] M. Sato, T. Hikihara, and T. Momoi, Field and temperature dependence of NMR relaxation rate in the magnetic quadrupolar liquid phase of spin- $\frac{1}{2}$ frustrated ferromagnetic chains, Phys. Rev. B 83, 064405 (2011).

[15] O. A. Starykh and L. Balents, Excitations and quasi-onedimensionality in field-induced nematic and spin density wave states, Phys. Rev. B 89, 104407 (2014).

[16] H. Onishi, Magnetic excitations of spin nematic state in frustrated ferromagnetic chain, J. Phys. Soc. Jpn. 84, 083702 (2015).

[17] S. C. Furuya, Angular dependence of electron spin resonance for detecting the quadrupolar liquid state of frustrated spin chains, Phys. Rev. B 95, 014416 (2017).

[18] N. Büttgen, K. Nawa, T. Fujita, M. Hagiwara, P. Kuhns, A. Prokofiev, A. P. Reyes, L. E. Svistov, K. Yoshimura, and M. Takigawa, Search for a spin-nematic phase in the quasi-onedimensional frustrated magnet $\mathrm{LiCuVO}_{4}$, Phys. Rev. B 90, 134401 (2014).

[19] A. Orlova, E. L. Green, J. M. Law, D. I. Gorbunov, G. Chanda, S. Krämer, M. Horvatić, R. K. Kremer, J. Wosnitza, and G. L. J. A. Rikken, Nuclear Magnetic Resonance Signature of the Spin-Nematic Phase in $\mathrm{LiCuVO}_{4}$ at High Magnetic Fields, Phys. Rev. Lett. 118, 247201 (2017).

[20] Y. Kono, T. Sakakibara, C. P. Aoyama, C. Hotta, M. M. Turnbull, C. P. Landee, and Y. Takano, Field-Induced Quantum Criticality and Universal Temperature Dependence of the Magnetization of a Spin- $\frac{1}{2}$ Heisenberg Chain, Phys. Rev. Lett. 114, 037202 (2015).

[21] S. Blundell, Magnetism in Condensed Matter, Oxford Master Series in Condensed Matter Physics 4 (Oxford University Press, Oxford, 2001).

[22] L. E. Svistov, T. Fujita, H. Yamaguchi, S. Kimura, K. Omura, A. Prokofiev, A. I. Smirnov, Z. Honda, and M. Hagiwara, New high magnetic field phase of the frustrated $S=1 / 2$ chain compound $\mathrm{LiCuVO}_{4}$, JETP Lett. 93, 21 (2011).

[23] G. Meunier, J. Darriet, and J. Galy, L'oxyde double $\mathrm{TeVO}_{4}$ II. Structure cristalline de $\mathrm{TeVO}_{4}-\beta$-relations structurales, J. Solid State Chem. 6, 67 (1973).

[24] Yu. Savina, O. Bludov, V. Pashchenko, S. L. Gnatchenko, P. Lemmens, and $\mathrm{H}$. Berger, Magnetic properties of the antiferromagnetic spin- $\frac{1}{2}$ chain system $\beta-\mathrm{TeVO}_{4}$, Phys. Rev. B 84, 104447 (2011).
[25] A. Saúl and G. Radtke, Density functional approach for the magnetism of $\beta-\mathrm{TeVO}_{4}$, Phys. Rev. B 89, 104414 (2014).

[26] M. Pregelj, A. Zorko, O. Zaharko, H. Nojiri, H. Berger, L. C. Chapon, and D. Arčon, Spin-stripe phase in a frustrated zigzag spin- $\frac{1}{2}$ chain, Nat. Commun. 6, 7255 (2015).

[27] Yu. O. Savina, A. N. Bludov, V. A. Pashchenko, S. L. Gnatchenko, Yu. V. Savin, S. Schäfer, P. Lemmens, and H. Berger, A study of the magnetic properties of a quasi-onedimensional magnet $\beta-\mathrm{TeVO}_{4}$ in the frame of the $J_{1}-J_{2}$ model, Low Temp. Phys. 41, 659 (2015).

[28] F. Weickert, N. Harrison, B. L. Scott, M. Jaime, A. Leitmäe, I. Heinmaa, R. Stern, O. Janson, H. Berger, H. Rosner et al., Magnetic anisotropy in the frustrated spin-chain compound $\beta$ - $\mathrm{TeVO}_{4}$, Phys. Rev. B 94, 064403 (2016).

[29] M. Pregelj, O. Zaharko, U. Stuhr, A. Zorko, H. Berger, A. Prokofiev, and D. Arčon, Coexisting spinons and magnons in frustrated zigzag spin- $\frac{1}{2}$ chain compound $\beta-\mathrm{TeVO}_{4}$, Phys. Rev. B 98, 094405 (2018).

[30] M. Pregelj, A. Zorko, M. Klanjšek, O. Zaharko, J. S. White, O. Prokhnenko, M. Bartkowiak, H. Nojiri, H. Berger, and D. Arčon, Magnetic ground state of the frustrated spin- $\frac{1}{2}$ chain compound $\beta-\mathrm{TeVO}_{4}$ at high magnetic fields, Phys. Rev. B 100, 094433 (2019).

[31] A. Singhania and S. Kumar, Temperature driven multiple phase transitions and quadrupolar order in $\beta-\mathrm{TeVO}_{4}$, Phys. Rev. B 101, 064403 (2020).

[32] See Supplemental Material at http://link.aps.org/supplemental/ 10.1103/PhysRevB.102.081104 for details of the NMR technique and of the isotopic enrichment of the sample by ${ }^{17} \mathrm{O}$.

[33] V. A. Klochan, N. M. Kovtun, G. A. Troitskij, and V. M. Khmara, Spin flip induced by an external magnetic field in erbium orthoferrite in the range of rare-earth ion ordering temperatures, Zh. Éksp. Teor. Fiz. 81, 627 (1981) [Sov. Phys. JETP 54, 335 (1981)].

[34] V. G. Bar'yachtar, A. E. Borovik, and V. A. Popov, Theory of the intermediate state of antiferromagnets, Zh. Éksp. Teor. Fiz. 62, 2233 (1972) [Sov. Phys. JETP 35, 1169 (1972)].

[35] V. G. Bar'yakhtar, A. N. Bogdanov, and D. A. Yablonskiǔ, The physics of magnetic domains, Sov. Phys. Usp. 31, 810 (1988).

[36] M. Pregelj, O. Zaharko, A. Zorko, M. Gomilšek, O. Sendetskyi, A. Günther, M. Ozerov, S. A. Zvyagin, H. Luetkens, C. Baines et al., Controllable broadband absorption in the mixed phase of metamagnets, Adv. Funct. Mater. 25, 3634 (2015).

[37] M. Horvatić, Magnetic nuclear spin-lattice relaxation in NMR of orthorhombic crystals in the presence of strong quadrupole coupling, J. Phys.: Condens. Matter 4, 5811 (1992).

[38] V. F. Mitrović, M.-H. Julien, C. de Vaulx, M. Horvatić, C. Berthier, T. Suzuki, and K. Yamada, Similar glassy features in the ${ }^{139} \mathrm{La}$ NMR response of pure and disordered $\mathrm{La}_{1.88} \mathrm{Sr}_{0.12} \mathrm{CuO}_{4}$, Phys. Rev. B 78, 014504 (2008).

[39] B. Willenberg, M. Schäpers, A. U. B. Wolter, S.-L. Drechsler, M. Reehuis, J.-U. Hoffmann, B. Büchner, A. J. Studer, K. C. Rule, B. Ouladdiaf, S. Süllow, and S. Nishimoto, Complex Field-Induced States in Linarite $\mathrm{PbCuSO}_{4}(\mathrm{OH})_{2}$ with a Variety of High-Order Exotic Spin-Density Wave States, Phys. Rev. Lett. 116, 047202 (2016).

[40] L. Heinze, G. Bastien, B. Ryll, J.-U. Hoffmann, M. Reehuis, B. Ouladdiaf, F. Bert, E. Kermarrec, P. Mendels, S. Nishimoto 
et al., Magnetic phase diagram of the frustrated spin chain compound linarite $\mathrm{PbCuSO}_{4}(\mathrm{OH})_{2}$ as seen by neutron diffraction and ${ }^{1} \mathrm{H}-\mathrm{NMR}$, Phys. Rev. B 99, 094436 (2019).

[41] M. Pregelj, A. Zorko, M. Gomilšek, M. Klanjšek, O. Zaharko, J. S. White, H. Luetkens, F. Coomer, T. Ivek, D. R. Góngora, H. Berger, and D. Arčon, Elementary excitation in the spinstripe phase in quantum chains, npj Quantum Mater. 4, 22 (2019).
[42] R. O. Kuzian and S. L. Drechsler, Exact one- and twoparticle excitation spectra of acute-angle helimagnets above their saturation magnetic field, Phys. Rev. B 75, 024401 (2007).

[43] S. Nishimoto, S.-L. Drechsler, R. Kuzian, J. Richter, and J. van den Brink, Interplay of interchain interactions and exchange anisotropy: Stability and fragility of multipolar states in spin- $\frac{1}{2}$ quasi-one-dimensional frustrated helimagnets, Phys. Rev. B 92, 214415 (2015). 